\title{
Direct observation of spin-wave focusing by a Fresnel lens
}

\author{
Joachim Gräfe $\odot,{ }^{1,}{ }^{*}$ Pawel Gruszecki, ${ }^{2,8}$ Mateusz Zelent, ${ }^{2}$ Martin Decker, ${ }^{3}$ Kahraman Keskinbora, ${ }^{1}$ Matthias Noske, ${ }^{1}$ \\ Przemysław Gawronski, ${ }^{4}$ Hermann Stoll,,${ }^{1,5}$ Markus Weigand, ${ }^{1,6}$ Maciej Krawczyk, ${ }^{2}$ Christian H. Back, ${ }^{3,7}$ \\ Eberhard J. Goering, ${ }^{1}$ and Gisela Schütz ${ }^{1}$ \\ ${ }^{1}$ Max Planck Institute for Intelligent Systems, Stuttgart, Germany \\ ${ }^{2}$ Faculty of Physics, Adam Mickiewicz University, Poznan, Poland \\ ${ }^{3}$ Department of Physics, University of Regensburg, Regensburg, Germany \\ ${ }^{4}$ AGH University of Science and Technology, Krakow, Poland \\ ${ }^{5}$ Institute of Physics, Johannes Gutenberg University, Mainz, Germany \\ ${ }^{6}$ Helmholtz-Zentrum Berlin für Materialien und Energie GmbH, Berlin, Germany \\ ${ }^{7}$ Technical University Munich, Munich, Germany \\ ${ }^{8}$ Institute of Molecular Physics, Polish Academy of Science, Poznan, Poland
}

(Received 30 August 2017; revised 9 April 2020; accepted 8 June 2020; published 15 July 2020)

\begin{abstract}
Spin waves are discussed as promising information carrier for beyond complementary metal-oxide semiconductor data processing. One major challenge is guiding and steering of spin waves in a uniform film. Here, we explore the use of diffractive optics for these tasks by nanoscale real-space imaging using $\mathrm{x}$-ray microscopy and careful analysis with micromagnetic simulations. We discuss the properties of the focused caustic beams that are generated by a Fresnel-type zone plate and demonstrate control and steering of the focal spot. Thus, we present a steerable and intense nanometer-sized spin-wave source. Potentially, this could be used to selectively illuminate magnonic devices like nano-oscillators.
\end{abstract}

DOI: 10.1103/PhysRevB.102.024420

\section{INTRODUCTION}

Magnonics, i.e., the artificial manipulation of spin waves, is a flourishing field of research with many potential uses in data processing within reach [1-4]. Apart from the technological applications the possibility to directly influence and observe these types of waves is of great interest in fundamental research. Guidance and steering of spin waves have been previously shown $[5,6]$ and lateral spin-wave confinement has been achieved $[7,8]$, e.g., by lateral restriction of the propagation medium [8] and self-collimation [9]. A main aspect of this confinement at relatively low frequencies is the formation of a caustic beam [10-12]. Additionally, it has been shown that refractive spin-wave optics can be realized [13-19].

Here, we explore the properties of diffractive optics for spin waves [20-22]. In principle, these diffractive optics have been shown to be able to focus spin waves [23-25]. A Fresnel zone plate is such a diffractive lens that is widely used in focusing of light [26]. As an array of holes acts as a diffraction lattice for spin waves [21], a hole arrangement seems to be a promising base for the realization of a diffractive lens. Here, we realize this idea for spin waves by using an alternating

*graefe@is.mpg.de

Published by the American Physical Society under the terms of the Creative Commons Attribution 4.0 International license. Further distribution of this work must maintain attribution to the author(s) and the published article's title, journal citation, and DOI. Open access publication funded by the Max Planck Society. arrangement of material (transmissive) and holes (absorbing), as shown in Fig. 1. We discuss spin-wave focusing utilizing this type of Fresnel zone plate lens. Using x-ray microscopy, we are able to directly image the propagation of spin waves into the nanometer-sized focal spot. Subsequently, we explore the operation mechanism of the magnonic Fresnel zone plate by micromagnetic simulation.

\section{METHODS AND THEORY}

\section{A. Spin-wave dispersion theory}

Spin-wave (SW) propagation, even in uniformly in-plane magnetized thin films, is complex and intrinsically anisotropic [27]. The dispersion relation that is wave-vector angledependent reads as [13,27]

$$
\begin{aligned}
\omega^{2}= & \gamma^{2} \mu_{0}^{2}\left\{H+l_{\mathrm{ex}}^{2} M_{\mathrm{S}} k^{2}-M_{\mathrm{S}}\left[1-f\left(k L_{z}\right)\right]\right\} \\
& \times\left\{H+l_{\mathrm{ex}}^{2} M_{\mathrm{S}} k^{2}-M_{\mathrm{S}} f\left(k L_{z}\right) \sin ^{2} \theta_{\mathbf{k}}\right\},
\end{aligned}
$$

where $\omega=2 \pi f$ is the angular frequency of SWs, $f$ is the frequency, $\mu_{0}$ is the permeability of vacuum, $H$ is the bias magnetic field, $M_{\mathrm{S}}$ is the saturation magnetization, $l_{\mathrm{ex}}=$ $\sqrt{\frac{2 A}{\mu_{0} M_{\mathrm{S}}^{2}}}$ is the exchange length, $A$ is the exchange constant, $L_{z}$ is the film's thickness, $k$ is the wave number, $\theta_{k}$ is the in-plane angle between the wavevector and the direction of the bias magnetic field, and $f(x)=1-[1-\exp (-x)] / x$.

For frequencies of magnetostatic modes, characterized by the strong dipolar interactions dominating over the isotropic exchange interactions, the difference of wavelengths 


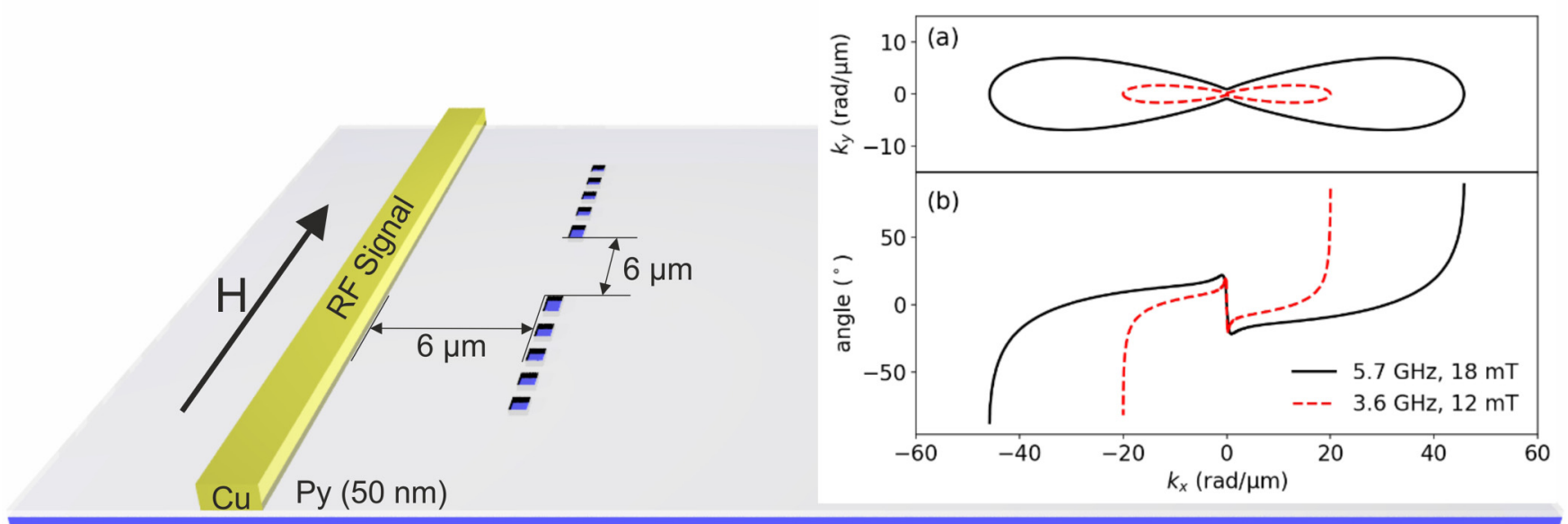

$\operatorname{SiN}(100 \mathrm{~nm})$

FIG. 1. Sketch of the magnonic zone plate samples for x-ray microscopy. $50 \mathrm{~nm}$ of Py are deposited on top of an $\mathrm{x}$-ray transparent $\mathrm{Si}_{3} \mathrm{~N}_{4}$ membrane. For $\mathrm{rf}$ excitation a 1.6- $\mu \mathrm{m}$-wide $\mathrm{Cu}$ stripline is deposited on top of the magnetic thin film as excitation source. The Fresnel zone plate is realized by an arrangement of holes at a distance of $6 \mu \mathrm{m}$ from the excitation source. Additionally, an in-plane magnetic bias field is applied along the stripline. (a) shows the isofrequency contour and (b) shows the angle of the group velocity with respect to the bias field in this film for $3.6 \mathrm{GHz} / 12 \mathrm{mT}$ (dashed red lines) and $5.7 \mathrm{GHz} / 18 \mathrm{mT}$ (solid black lines).

propagating in the orthogonal directions can be surprisingly significant. Isofrequency contours, i.e., slices of the twodimensional dispersion relation $f\left(k_{x}, k_{y}\right)$ for a given frequency, can be useful to visualize this anisotropy. Two isofrequency contours for spin waves in 50-nm-thick Permalloy film magnetized along the $x$ axis are shown in Fig. 1(a). These curves were obtained for two sets of frequencies and bias magnetic fields, viz., $3.6 \mathrm{GHz} / 12 \mathrm{mT}$ and $5.7 \mathrm{GHz} / 28 \mathrm{mT}$. A peanutlike shape of these contours confirms strong wavelength dependence on the direction of wave vector $\left(\theta_{\mathbf{k}}\right)$. For instance, for the 5.7-GHz case, the wavelengths of spin waves propagating along the direction of the magnetic bias field ( $y$ axis), so-called backward volume modes, is equal to 137 $\mathrm{nm}$ whereas, for the perpendicular to the field propagation, so-called Damon-Eshbach modes, the wavelength reaches 6.7 $\mu \mathrm{m}-\mathrm{a} 49$-fold difference. In the case of spin waves at 3.6 $\mathrm{GHz}$, the wavelengths equal $314 \mathrm{~nm}$ and $40 \mu \mathrm{m}$ for backward volume and Damon-Eshbach modes, respectively.

There is also an additional striking consequence of these peanut-shape-like isofrequency contours, viz., its influence on the energy flow. The angle of the group velocity $\mathbf{v}_{\mathrm{gr}}=\left[v_{x}, v_{y}\right]=\left[\frac{\partial \omega}{\partial k_{x}}, \frac{\partial \omega}{\partial k_{y}}\right]$ with respect to the bias field (applied along the $x$ axis) can be expressed as $\theta_{\mathrm{gr}}(\mathbf{k})=$ $\arctan \left(v_{x} / v_{y}\right)=\arctan \left(d k_{x} / d k_{y}\right)$. Hence, for regions with zero curvature $\partial^{2} k_{y} / \partial k_{x}^{2}=0$, the direction of the group velocity is the same for waves having different wave vectors. This occurs for wave vectors in regions of the isofrequency contour with zero curvature. Consequently, this allows the existence of very narrow caustic beams with low divergence that can even be emitted by a point source $[11,28,29]$. However, even for isofrequency contours with near-zero curvature $\frac{\partial^{2} k_{y}}{\partial k_{x}^{2}} \approx 0$, the direction of energy flow for a relatively broad range of wave vectors can be similar, therefore supporting energy flow in the form of causticlike beams.

This is clearly visible for the two sets of frequencies and bias fields discussed previously [cf. Fig. 1(a)]. The corresponding $\theta_{\mathrm{gr}}(\mathbf{k})$ dependence on the tangential to the field component of the wave vector is shown in Fig. 1(b). Similar angles of group velocity propagation are seen, for a broad range of wave vectors; e.g., for $5.7 \mathrm{GHz}$, this angle spans the range from $-10^{\circ}$ to $-17^{\circ}$ for approximately one-third of all available positive $k_{x}$ values. Similarly, for the 3.6$\mathrm{GHz}$ case, this angle spans the range from $-4^{\circ}$ to $-10^{\circ}$ for approximately one-third of all available positive $k_{x}$ values.

\section{B. Experimental details}

The focusing properties of the proposed lens design were initially estimated using a one-dimensional version of the Rayleigh-Sommerfeld approximation of scalar wave diffraction [30], which is widely used for photons [31,32]. In these initial estimates, the wavelength of Damon-Eshbach-type spin waves was assumed to be similar to those previously observed, thus neglecting the anisotropic dispersion properties of spin waves, but resulting in a sufficient design approximation to operate as proof of concept of a spin-wave lens.

50-nm-thick Permalloy film (Py, $\mathrm{Ni}_{80} \mathrm{Fe}_{20}$ ) films were deposited on $\mathrm{Si}_{3} \mathrm{~N}_{4}(100 \mathrm{~nm}) / \mathrm{Si}(100)$ substrates by evaporation at a base pressure better than $1 \times 10^{-7} \mathrm{mbar}$ and covered by $5-\mathrm{nm}$ thin Al. The holes making up the zone plates were structured by e-beam lithography and lift-off. Subsequently, $10 \mathrm{~nm}$ of $\mathrm{Al}_{2} \mathrm{O}_{3}$ were deposited by atomic layer deposition for isolation of a 1.6- $\mu \mathrm{m}$-wide microstrip antenna $[\mathrm{Cr}(10$ $\mathrm{nm}) / \mathrm{Cu}(150 \mathrm{~nm}) / \mathrm{Al}(5 \mathrm{~nm})]$ that was deposited on top. The overall sample design is schematically illustrated in Fig. 1.

Time-resolved scanning x-ray transmission microscopy (STXM) measurements were conducted at the Max Planck Institute for Intelligent Systems-operated MAXYMUS end station at the UE46-PGM2 beam line at the BESSY II synchrotron radiation facility. The samples were illuminated under perpendicular incidence by circularly polarized light in an applied in-plane field of up to $240 \mathrm{mT}$ that was generated by a set of four rotatable permanent magnets [33]. The photon 
energy was set to the absorption maximum of the $\mathrm{Fe} L_{3}$ edge to get optimal X-ray Magnetic Circular Dichroism (XMCD) contrast for imaging. A lock-in-like detection scheme allows sample excitation at arbitrary frequencies at a time resolution of 50 ps using all photons emitted by the synchrotron.

\section{Simulation details}

The micromagnetic simulations were performed using MUMAX3 with absorbing boundary layers along all edges of the film [34-37]. The studied system was discretized uniformly by $6 \times 6 \times 12.5-\mathrm{nm}^{3}$ unit cells. In simulations, a saturation magnetization of $M_{s}=0.8 \times 10^{6} \mathrm{~A} / \mathrm{m}$, an exchange constant of $A=1.3 \times 10^{-11} \mathrm{~J} / \mathrm{m}$, and a damping constant $\alpha=0.001$ were used. First, the static magnetization configuration was stabilized for a given magnetic field ( $22 \mathrm{mT}$ applied along the grating if not written differently); then, the spin waves were excited by a continuous harmonic signal of small amplitude at $5.7 \mathrm{GHz}$ at a distance of $1.5 \mu \mathrm{m}$ from the zone. To illustrate the flow of spin-wave energy after transmission through this zone, we calculated the power of spin waves as the sum of squares of perpendicular magnetization component after 20 periods of spin-wave propagation.

\section{RESULTS AND DISCUSSION}

\section{A. Focusing characteristics}

Spin waves passing through such a Fresnel zone plate were imaged by time-resolved STXM with XMCD [38] contrast. This technique visualizes the spatial distribution of the normal component of the time-dependent magnetization of the sample [39]. The spin-wave amplitude derived from these data is shown in Fig. 2 (cf. Refs. [40] and [41], Supplemental Mate- rial, for spin-wave movies at 3.6 and $5.7 \mathrm{GHz}$, respectively). While Fig. 2(a) shows the spin-wave amplitude at $3.6 \mathrm{GHz}$, this lens geometry is also capable of focusing spin waves at $5.7 \mathrm{GHz}$ [shown in Fig. 2(b)], indicating that this type of lens is capable of operating in a broad frequency range. Areas with high spin-wave amplitude are shown in bright colors, and areas with low amplitude are shown in black.

Here, an amplitude increase of $23 \%$ at a focal distance of $6 \mu \mathrm{m}$ behind the lens is found, thus overcompensating the spin-wave damping during propagation in Permalloy. This enhancement is accompanied by the confinement of the spinwave beam waist to a $840-\mathrm{nm}$ full width at half maximum (FWHM). In the focal plane, the focal spot features a fivefold increase in amplitude within the central region behind the zone plate. Furthermore, the focal spot shows an amplitude increase by a factor of 2 compared to the whole interference pattern. Although this zone plate design only features a limited number of slits, this clearly shows that focusing of spin waves is possible, and the focal spot has significantly higher spin-wave intensity than the surrounding spin-wave landscape.

Similar behavior is found at a higher frequency of 5.7 GHz. At these frequencies, the focal length is reduced to $4.5 \mu \mathrm{m}$ [shown in Fig. 2(b)]. Compared to lower frequencies, the focal width is slightly increased to 1.1- $\mu \mathrm{m}$ FWHM in our measurements. Also, in this case, there is a substantial enhancement of the spin-wave amplitude in the focal spot within the central region behind the zone plate, and the focal spot stands out of the whole interference pattern behind the zone plate, resulting in the most intense spot in the focal plane.

Furthermore, the spin-wave damping during propagation was approximated by fitting an exponential decay to the

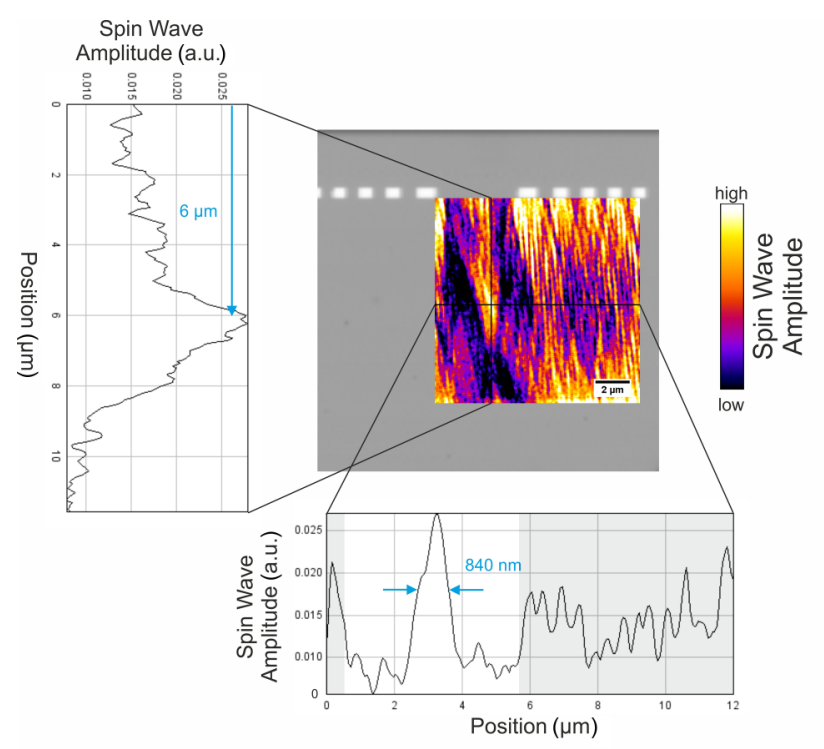

(a)

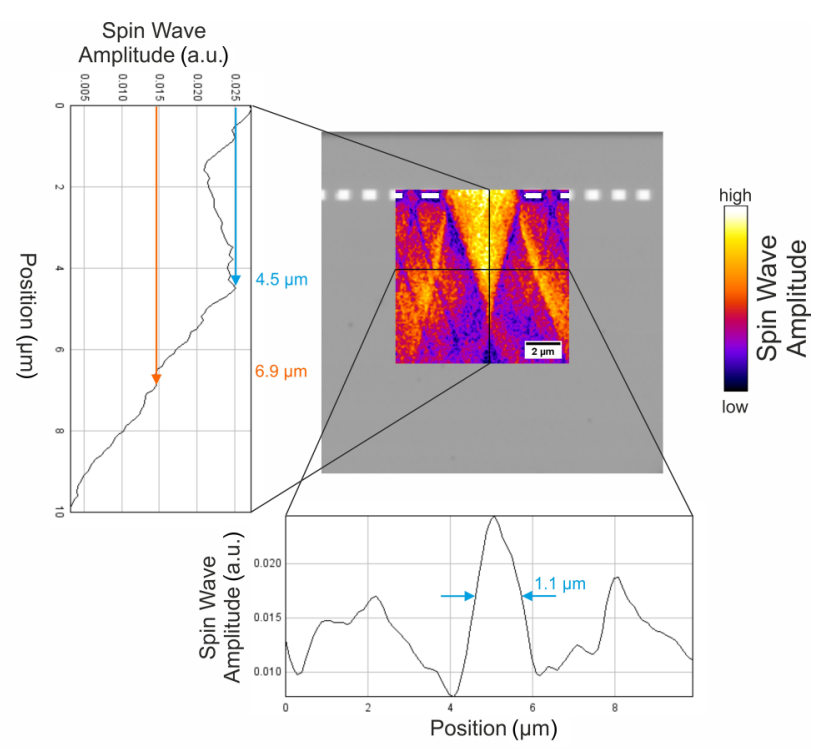

(b)

FIG. 2. Spin-wave amplitude for transmission through a hole-based Fresnel zone plate in a 50-nm Py thin film (a) at 3.6 GHz with an applied in-plane bias field of $-12 \mathrm{mT}$ and (b) at $5.7 \mathrm{GHz}$ with an applied in-plane bias field of $-18 \mathrm{mT}$. Additionally, cuts through the amplitude along the propagation direction and through the focal plane are shown. For (a) the focal spot is found $6 \mu \mathrm{m}$ behind the lens and the spin waves are confined to 840-nm FWHM. Furthermore, the amplitude is increased by $23 \%$, thus overcompensating for damping during spin-wave propagation. 


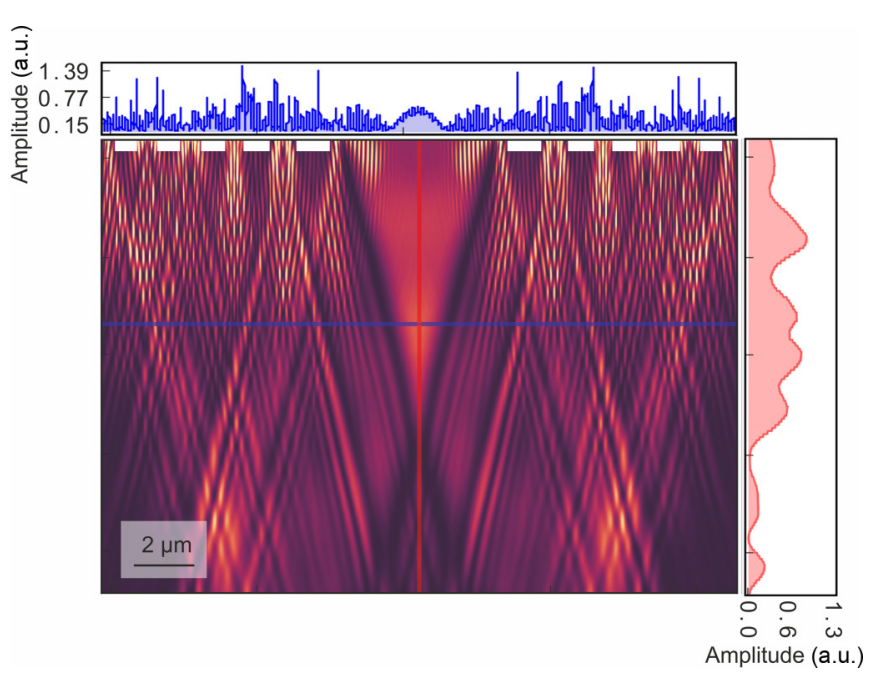

FIG. 3. Simulation of the spin-wave amplitude for transmission through a hole-based Fresnel zone plate in a 50-nm Py thin film at $5.7 \mathrm{GHz}$ with an applied in-plane bias field of $22 \mathrm{mT}$. Qualitatively, the same spin-wave pattern as in the experiment (cf. Fig. 2) is found. Furthermore, a comparable focus distance and size is found.

overall spin-wave amplitude along the propagation direction. When considering damping, an amplitude enhancement of $80 \%$ is reached in the focal spot in comparison to the source. This is equal to the amplitude enhancement that is calculated for light in our simplified approach and also compares well to the expectation for a caustic beam [10,31,42]. However, the simplified approach for photons fails to describe the focal length correctly, because the anisotropic dispersion of spin waves in a uniform medium is neglected [3].

Therefore, the system has to be modeled by micromagnetic simulations, which is shown in Fig. 3. The micromagnetic simulation shows a very good qualitative agreement with the experiment. The beam pattern from the experiment is recovered, including the short-wavelength ripples perpendicular to the propagation direction. Furthermore, for the focal length fair agreement between the experiment and simulation is found with 6 and $6.9 \mu \mathrm{m}$, respectively. This mismatch can be attributed to a slight field mismatch and the fact that the experiment shows a focal spot that is off the center axis, which is discussed later.

Another peculiarity is the small focal waist in comparison to the wavelength of several $\mu \mathrm{m}$ of the Damon-Eshbach (DE, $\vec{k} \perp \vec{M}$ )-type spin waves observed here. In principle the focal width is significantly smaller than the wavelength of the spin waves that are being focused. However, this is not real subwavelength focusing due to the above-mentioned anisotropic dispersion relation and can be explained by the formation of caustic beams as discussed in the literature $[3,11]$. The wavelength in the $x$ direction, i.e., backward volume (BV, $\vec{k} \|$ $\vec{M})$ geometry, is significantly shorter at the same frequency. Thus, the focal width is not smaller than the wavelength along the focal line. This small wavelength in BV direction is also the source of the amplitude ripples perpendicular to the propagation direction. As spin waves are scattered at the nonuniform magnetization structure around the holes, BV spin waves are excited and a standing spin-wave pattern forms between the holes. This shows the importance of the local magnetization landscape around the holes that is discussed in the following.

\section{B. Diffraction of spin waves in nonuniform magnetization landscapes}

In the following, we discuss the analytical description of spin waves scattered in nonuniform magnetization landscapes to understand the experimental and micromagnetic simulation results described above. In general, a complex profile $m(x, y)$ of waves diffracted at a slit can be described by the integral

$$
m(x, y) \propto \int_{-\infty}^{\infty} a\left(x^{\prime}\right) s\left(x-x^{\prime}, y-y_{0}\right) d x^{\prime},
$$

where $a(x)=|a(x)| \exp [i \varphi(x)]$ is the aperture function, $y_{0}$ is the location of the interface along which integration takes place, and $s(x, y)$ is the complex profile of waves emitted by an ideal point source. The function $s(x, y)$ can be derived analytically or numerically. It is noteworthy that Eq. (2) states that any point at the plane $y=y_{0}$ is a point source of waves emitting them with the amplitude and phase described by the aperture function $a(x)$. This equation describes the Huygens-Fresnel principle for a textbook isotropic medium (with $s \propto \exp [i \mathbf{k} \cdot \mathbf{r}]$ and $|\mathbf{k}|=k_{0}$ ). However, in the case of dipolar spin waves, their dispersion is strongly anisotropic, and, therefore, the profile of $s(x, y)$ is more complex [11]. Thus, we use a simulated $s(x, y)$; however, in principle, it also can be calculated analytically [11]. Namely, we have simulated profile of SWs emitted by a point source using MUMAX3 [35] and then processed it to the complex form, and subsequently, numerically solved the integral equation (2).

Let us consider the diffraction of 5.7-GHz spin waves at the 6- $\mu \mathrm{m}$-long and 550-nm-wide slit. The most straightforward way to model this slit is the aperture function being the rectangle function of length equal to the width of the slit, i.e., $a(x)=$ $\operatorname{Rect}\left(\frac{x}{6 \mu \mathrm{m}}\right)$, cf. Fig. 4(a). The real part spin-wave profile calculated by Eq. (2) for this aperture is displayed in Fig. 4(b). Additionally, the full micromagnetic simulation of spin waves diffracted at that slit is presented in Fig. 4(c) as comparison. The rectangular holes and the slit are schematically indicated by the black and white rectangles above the plot, respectively. It is visible that a simple representation of the aperture by the rectangle functions reproduces most of the features of the spin-wave profile. However, several aspects can be extracted from a more detailed analysis. Firstly, simulations predict a nonzero transmission of spin waves through the air gap due to the dipolar coupling. Secondly, an approximately $90^{\circ}$ phase shift between long-wavelength Damon-Eschbach spin-wave modes transmitted through the slit and the air gap is present (not visible in the intensity plots in Fig. 4). And thirdly, the V-shaped causticlike beam jets are shifted away from the interface by approximately $600 \mathrm{~nm}$ and to the center of the slit by approximately $250 \mathrm{~nm}$, which was also described by Schneider et al. observed in Ref. [11]. This can be clearly seen in the zoomed-in region near the edge of the slit shown as an inset in Fig. 4(c).

Consequently, in order to reproduce these features, we have assumed the most straightforward possible aperture function, i.e., $|a(x)|=0.51+0.49 \operatorname{Rect}\left(\frac{x}{5.5 \mu \mathrm{m}}\right)$ and $\varphi(x)=$ 


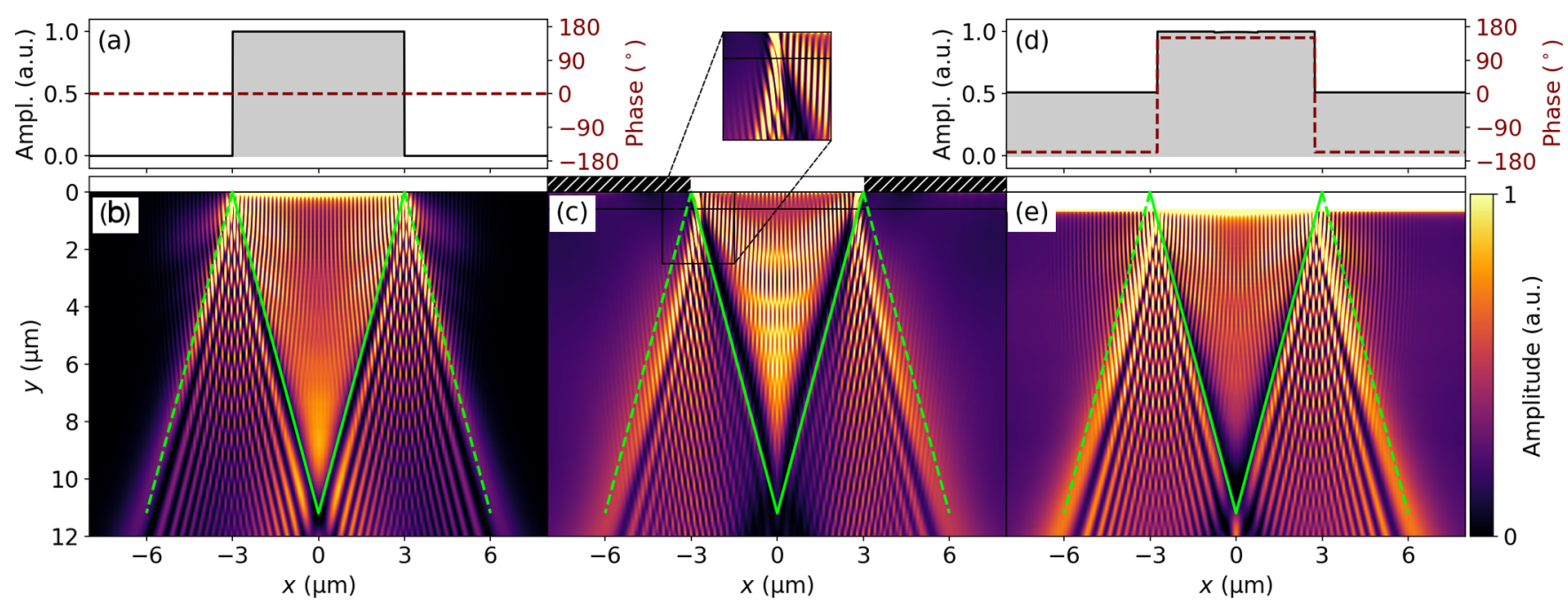

FIG. 4. (a), (d) The aperture functions $a(x)$ used in calculations. The solid black line represents the amplitude of the aperture function $|a(x)|$, whereas the dashed red line denotes its phase $\varphi(x)$. (a) Presents function $a(x)=\operatorname{Rect}\left(\frac{x}{6 \mu \mathrm{m}}\right)$, whereas (b) displays $a(x)=$ $\left[0.51+0.49 \operatorname{Rect}\left(\frac{x}{5.5 \mu \mathrm{m}}\right)\right] \times \exp \left[i\left(-150^{\circ}-90^{\circ} \operatorname{Rect}\left(\frac{x}{5.5 \mu \mathrm{m}}\right)\right)\right]$. (b), (e) Profile of spin waves at frequency $5.7 \mathrm{GHz}$ calculated with the application of the apertures shown in (a) and (d), respectively. The spin-wave profile in (e) is obtained for the interface located at $y=$ $600 \mathrm{~nm}$. (c) Results of micromagnetic simulations of the diffraction of spin waves at frequency $5.7 \mathrm{GHz}$ at the 6- $\mu \mathrm{m}-\mathrm{long}$ slit. The inset shows the zoomed-in region marked by the hatched black square, whereas the horizontal black line represents the location where the beam splits. The black solid horizontal lines in (c) and (e) represent the position of the interface used in calculations of the profile presented in (e). Green lines indicate the dominating direction of the group velocities along which spin-wave fronts align (viz., $\pm 15^{\circ}$ with respect to the $y$ axis).

$-150^{\circ}-90^{\circ} \operatorname{Rect}\left(\frac{x}{5.5 \mu \mathrm{m}}\right)$, cf. Fig. 4(d). Moreover, we have shifted the interface $600 \mathrm{~nm}$ apart from the interface [cf. the horizontal black lines in Fig. 4(c) and (d). The calculated spin-wave profile being displayed in Fig. 4(e), indeed, shows excellent agreement with micromagnetic simulations. The question that arises here is the origin of shift of the jets of causticlike beams emitted at the sides of the slit. In order to answer this question, we analyze the magnetic configuration and static demagnetizing field profile in the vicinity of the holes.
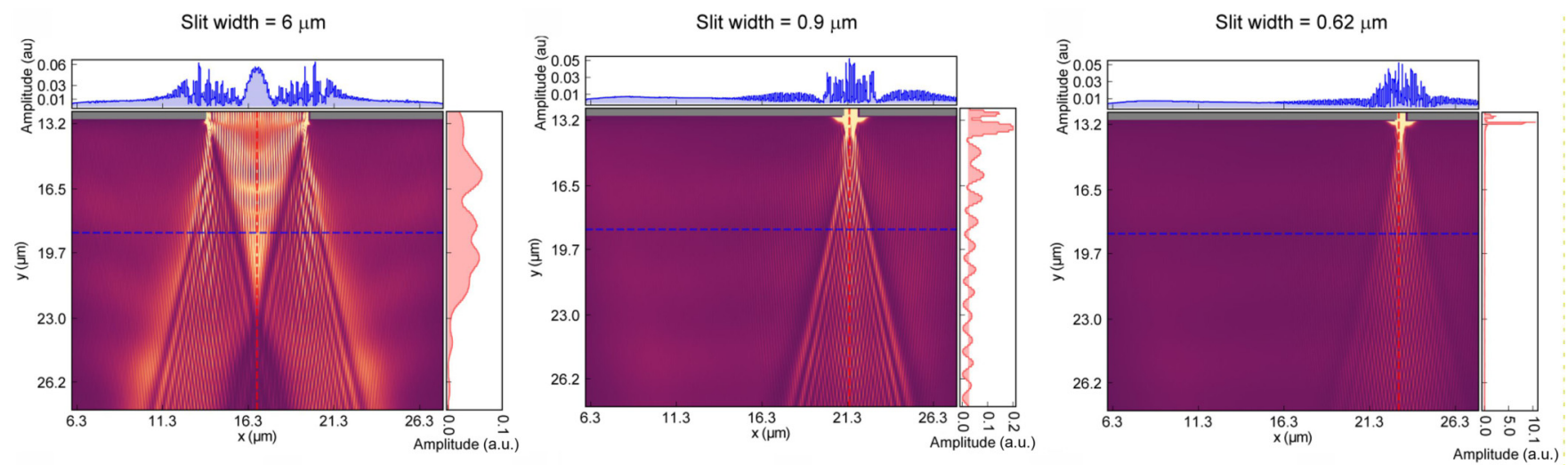

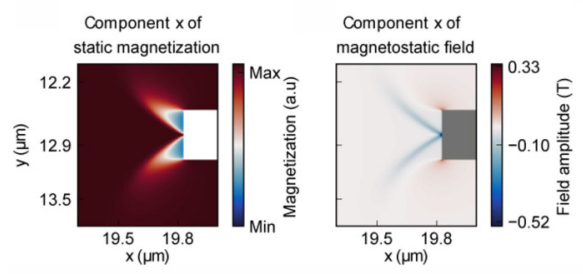

(a)

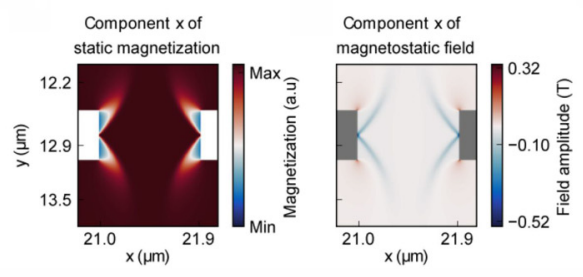

(b)
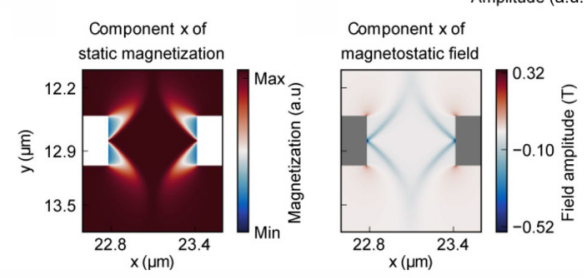

(c)

FIG. 5. Simulation of the magnetic behavior of the various slit widths (a) $6 \mu \mathrm{m}$, (b) $0.9 \mu \mathrm{m}$, and (c) $0.6 \mu \mathrm{m}$ that constitute the zone plate structure in a 50-nm-thin Py film at 5.7 GHz with an applied in-plane bias field of $22 \mathrm{mT}$ along the $x$ direction. For each slit the spin-wave transmission, the local static magnetization, and demagnetization fields are shown. It becomes evident that the magnetization is no longer independent when the slit width is reduced and the magnetostatic fields begin to overlap. 


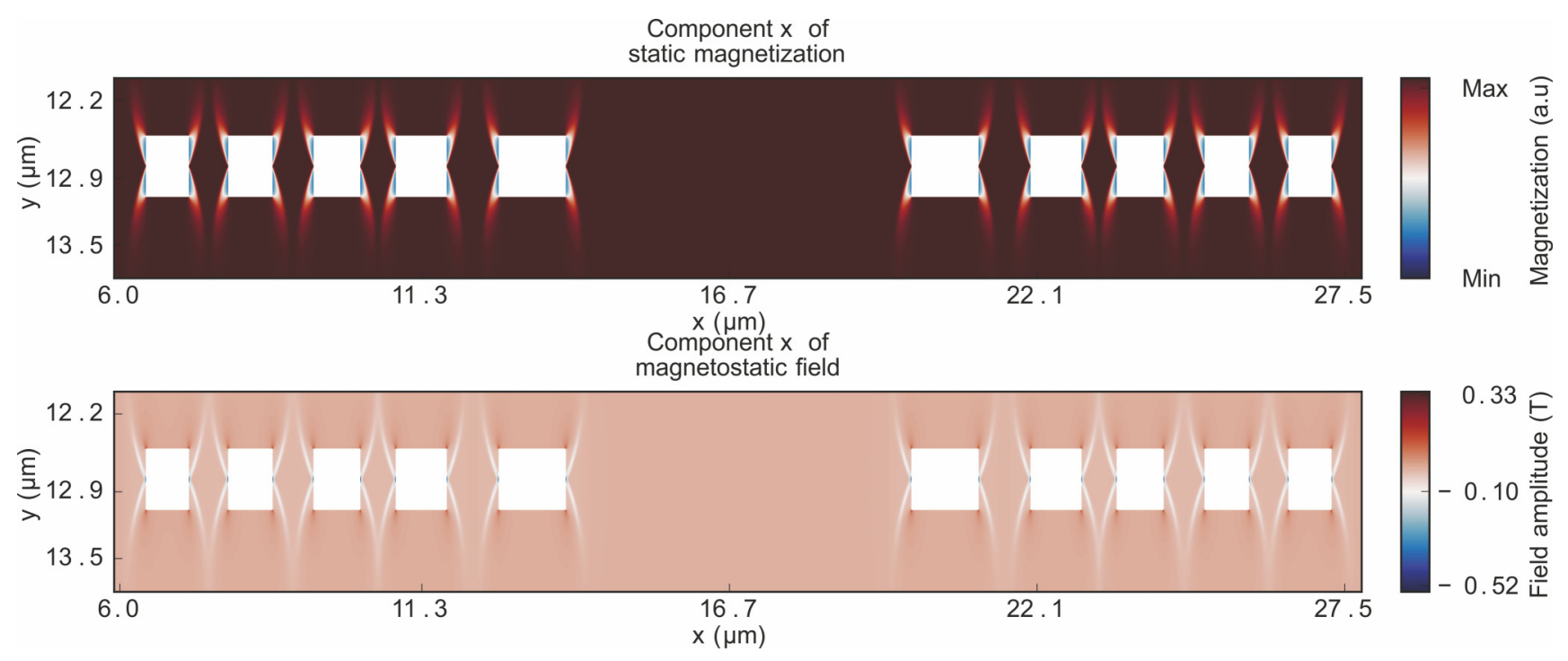

FIG. 6. Simulation of the static magnetization and the magnetostatic fields of the full zone plate structure in a 50-nm-thin Py film at $5.7 \mathrm{GHz}$ with an applied in-plane bias field of $22 \mathrm{mT}$ along the $x$ direction. While the magnetization in the central slit is mostly undisturbed, the influence area of the demagnetization fields in the outer slits overlap and form a complex magnetization landscape.

\section{Superposition of demagnetization fields}

To elucidate the contribution of the individual slits to the overall demagnetization field, Fig. 5 shows the dependence of the causticlike spin-wave beams emitted by slits with different sizes. The spin-wave amplitude after passing through the slit as well as the static magnetization configuration and the magnetostatic field are shown. From the simulation of the widest slit [cf. Fig. 5(a)] that corresponds to the central slit in the full zone plate, it becomes clear that the distorted magnetization landscape at the hole edge has an impact on the spin-wave pattern. Their nonuniform magnetization structure, due to demagnetization fields, extends significantly into the slit, and the area where the causticlike beam is formed is further constrained inwards from the edges of the hole.

When the slit size is reduced, as shown in Figs. 5(b) and 5(c), the magnetostatic fields generated from both holes overlap and a nonuniform magnetization structure is formed that extends significantly into the area behind the holes.
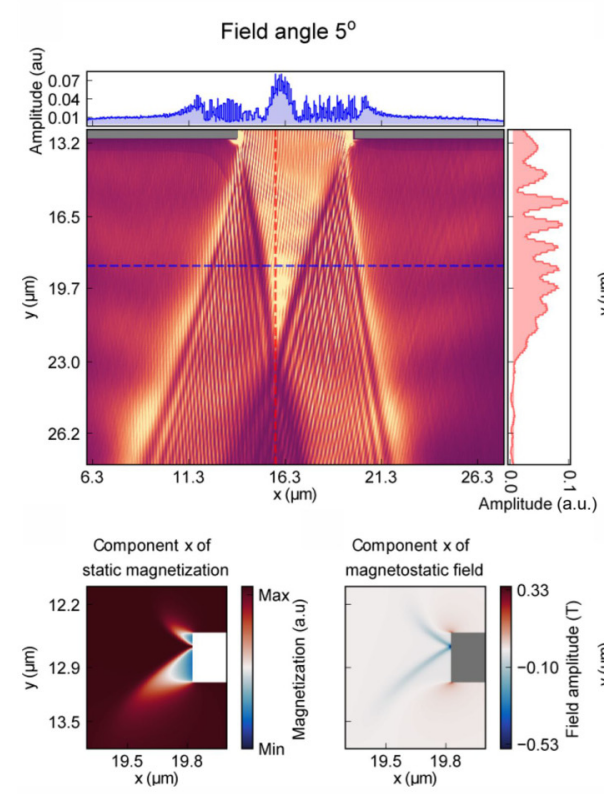

(a)
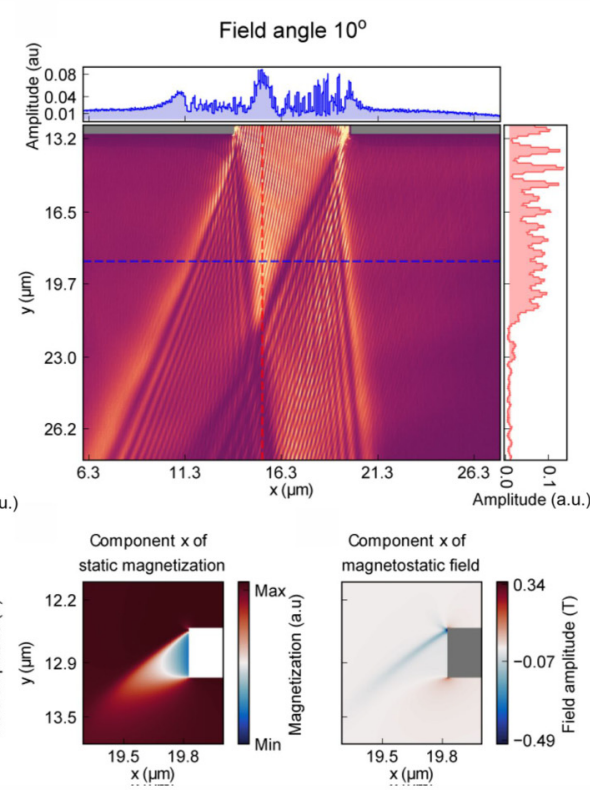

(b)
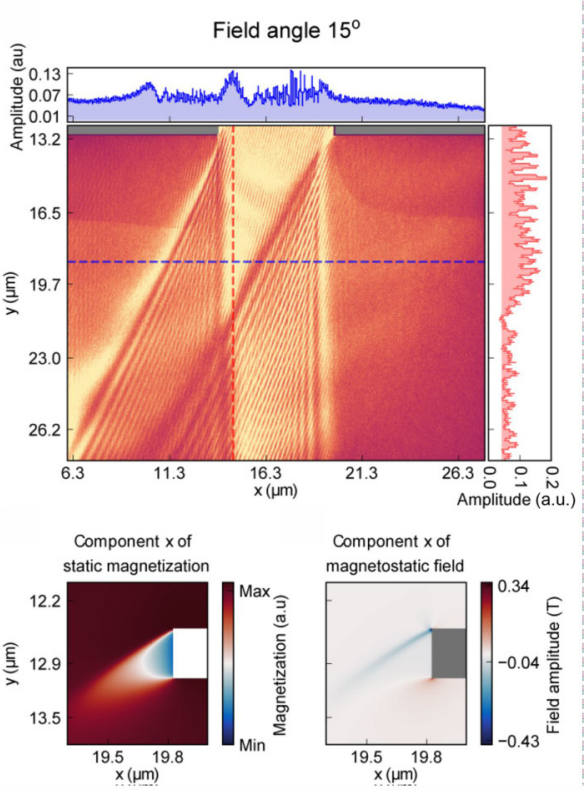

(c)

FIG. 7. Simulation of the magnetic behavior of the central slit of a zone plate structure in a 50-nm-thin Py film at $5.7 \mathrm{GHz}$ with an applied in-plane bias field magnitude of $22 \mathrm{mT}$ along the $x$ direction. The in-plane field is tilted to angles of (a) $5^{\circ}$, (b) $10^{\circ}$, and (c) $15^{\circ}$ with respect to the $x$ axis. For each angle, the spin-wave amplitude after passing through the slit, the static magnetization of the slit edge, and the corresponding local magnetostatic field are shown. 
This also becomes noticeable in the spin-wave pattern that is formed behind these slits. Behind these strongly confined slits, a different causticlike beam is observed. The beams that are emitted outwards have higher amplitude than the beam in the center of the slit. In the full zone plate design these outer slits also contribute intensity towards the center of the pattern.

It is clear that the overlap of the nonuniform magnetization structure is not limited to a single slit, but can also extend to adjacent slits, if they are located close enough. Therefore, we calculated the static magnetization structure and the magnetostatic field of the full zone plate structure, shown in Fig. 6. While the complex and overlapping magnetization structure can account for the spin-wave interference pattern and the difference with respect to a simple caustic beam, its symmetry cannot explain the experimentally observed focal shift off the central axis. Thus, we explored the influence of small tilt angles of the applied in-plane bias field with respect to the hole axis.

Figure 7 shows the dependence of the properties of the wide central slits on small in-plane tilt angles of the bias fields. For very small angles $\left[5^{\circ}\right.$, cf. Fig. 7(a)] the local magnetization is similar to the straight case, discussed before. However, the spin-wave pattern after transmission through this slit is already significantly distorted, although the majority of the spin-wave intensity is still found in the center of the simulation window. When increasing the tilt angle $\left[10^{\circ}\right.$ and $15^{\circ}$, cf. Figs. 7 (b) and 7(c)] the nonuniform magnetization structure around the hole is significantly pushed outside of the slit, also leading to stronger overlap between adjacent slits in the full zone plate case. This results in a shift of the vertex from which V-shaped causticlike beam jets propagate. The position of this vertex follows the profile of the demagnetization field around the hold. Consequently, strong bending of the spin-wave beam that propagates behind the slit occurs. In the following, we explore this feature to steer the focal spot of the zone plate structure.

\section{Focus steering}

Unlike zone plates for photons, the focal position of the zone plate for spin waves is not fixed for a given frequency. In fact, the focal spot can be moved within a $40-\mu \mathrm{m}^{2}$ area behind the zone plate by changing the magnitude and tilt angle of the applied static field, as shown in Fig. 8 for experiments performed at $5.7 \mathrm{GHz}$. At low [-6 mT, Fig 8(a)] and high [-40 mT, Fig. 8(h)] field amplitudes, no focusing occurs, while the focal position is shifted from the outer edge of the central area to its center [Figs. 8(b)-8(g)] at intermediate field amplitudes. The same behavior can be observed for opposing field signs with inverted symmetry.

This change of the wavelength and the propagation direction is easily observed in the phase contrast of Fig. 8. This phase information at high resolutions is uniquely available from x-ray microscopy measurements and allows the experimental explanation of the spin-wave zone plate properties. For spin waves with larger field values [Figs. $8(\mathrm{~d})-8(\mathrm{~g})]$ the focal length is long, and the focal spot lies roughly in the center behind the zone plate. On the other hand, for lower fields and shorter spin waves [Figs. 8(b) and 8(c)] the focal length is shorter, and the focal spot lies further outwards of the

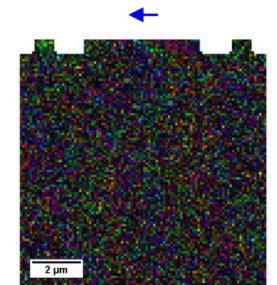

(a) $-6 \mathrm{mT}$

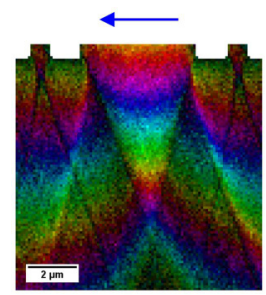

(d) $-16 \mathrm{mT}$

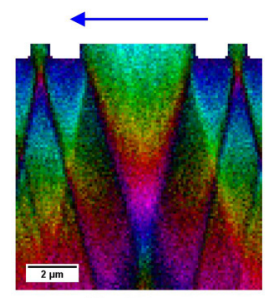

(f) $-28 \mathrm{mT}$

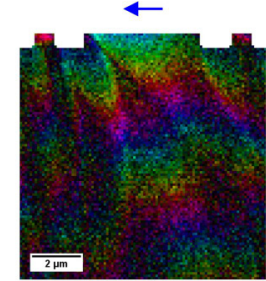

(b) $-8 \mathrm{mT}$
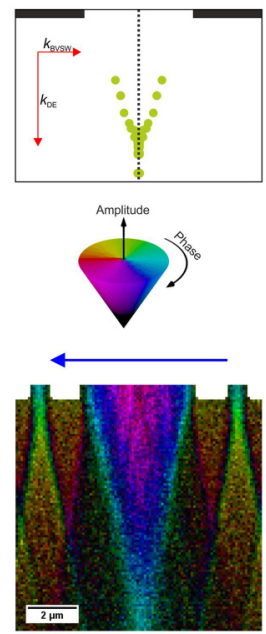

(g) $-36 \mathrm{mT}$

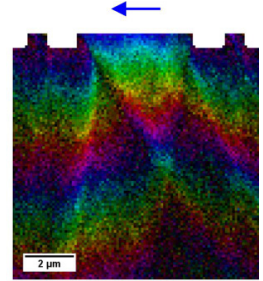

(c) $-10 \mathrm{mT}$

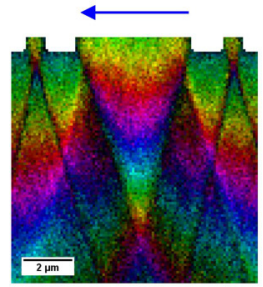

(e) $-22 \mathrm{mT}$

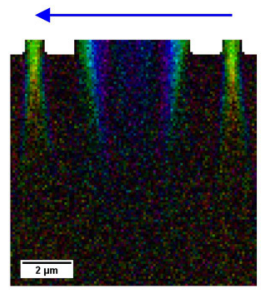

(h) $-40 \mathrm{mT}$
FIG. 8. Spin-wave amplitude and phase for transmission through a hole-based Fresnel zone plate in a 50-nm Py thin film at $5.7 \mathrm{GHz}$ with applied in-plane bias fields. At field magnitudes below $6 \mathrm{mT}$ (a) or above $40 \mathrm{mT}(\mathrm{h})$ no spin-wave focusing occurs. At intermediate fields the focal spot moves inwards and downwards with increasing Damon-Eshbach-type spin-wave contribution, i.e., the wave length increases and the emission angle turns perpendicular to the external field. The central inset shows the focal positions (maximum lateral confinement) as green dots for fields ranging from -8 to $-36 \mathrm{mT}$.

symmetry plane of the grating. Moreover, in Figs. 8(c)-8(e) the approximately $90^{\circ}$ phase shift between plane waves that propagated through the slit and through the holes, that we have predicted, is also visible.

In this context, the focusing cutoff at low- and high-field magnitudes can be understood as well.

At high fields $(-40 \mathrm{mT})$, only a beamlike propagation from the regions near the hole edges is present that is quickly damped out. We attribute that to the excitation being performed at a frequency lower than the ferromagnetic resonance frequency. Therefore, spin waves are localized only in the potential wells created by the demagnetizing field near the hole edges. For the subsequent measurements, performed at lower field magnitudes, we can see spin waves propagating through the whole measurement area. As expected, at higher field values, we find longer spin-wave lengths and the focal point is shifted further away from the zone plate. At low fields, on the other hand, there is a very shallow incidence angle of the spin waves on the zone plate. Here, the cutoff occurs when the spin waves cannot pass through the outermost slits and constructive beam formation is inhibited.

To better understand the influence of wave vectors constructing the resulting interference pattern and the in-plane 


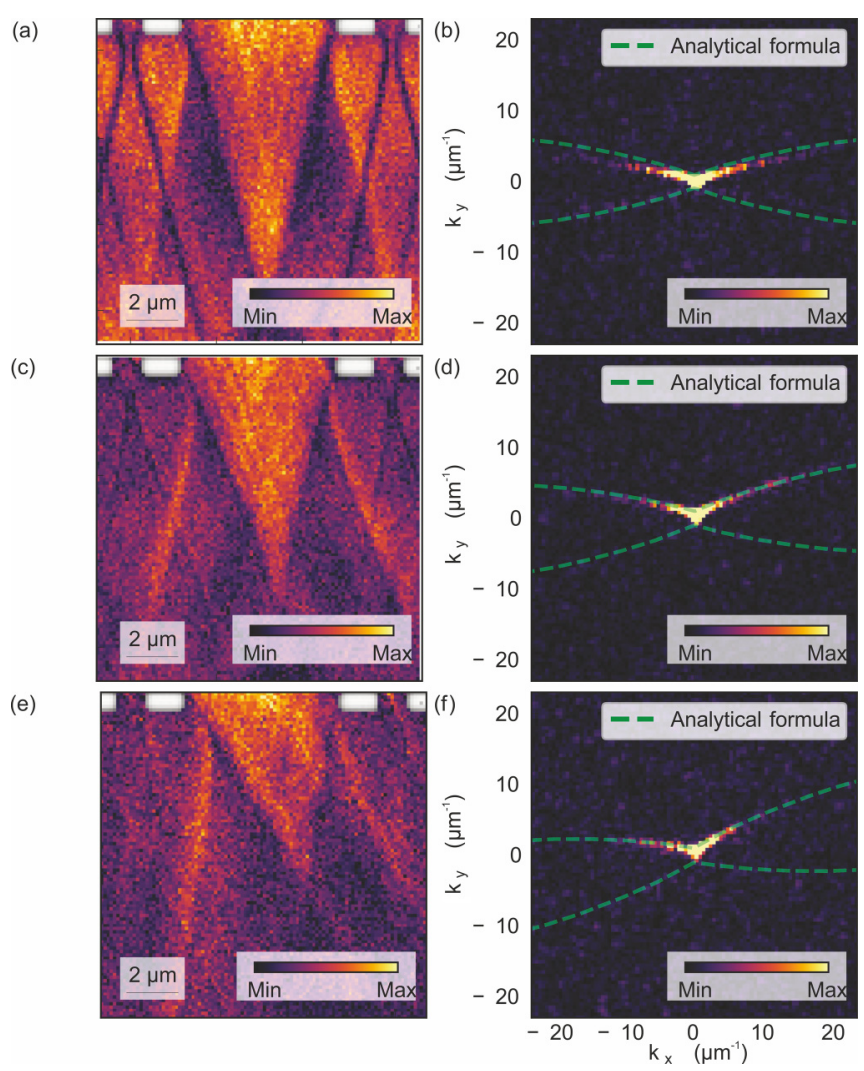

FIG. 9. Spin-wave amplitude in transmission through a holebased Fresnel zone plate in a 50-nm Py thin film at $5.7 \mathrm{GHz}$ with applied in-plane bias fields of (a) $-28 \mathrm{mT}$, (b) $-14 \mathrm{mT}$, and (c) -10 mT. Additionally, (b), (d), and (f) show the respective $k$-space transformations of the observed spin waves and the analytic isofrequency contour at the respective field geometries. From the isofrequency contour the tilt angle of the in-plane bias field can be determined to be $0^{\circ}, 3.5^{\circ}$, and $10^{\circ}$, respectively.

field angle, we transformed the real-space measurements into $k$ space by fast Fourier transformation. The resulting comparison of real-space and $k$-space maps is shown in Fig. 9 for $-28 \mathrm{mT}$ [Figs. 9(a) and 9(b)], $-14 \mathrm{mT}$ [Figs. 9(c) and 9(d)], and $-10 \mathrm{mT}$ [Figs. 9(e) and 9(f)]. Additionally, the analytical isofrequency contour for the given conditions is shown. The parameters for the analytical dispersion relation are determined by least-square fitting to the experimental data. Therefore, only the bias field angle with respect to the grating was a free parameter and used to determine the tilt angle in the experiment to be $0^{\circ}, 3.5^{\circ}$, and $10^{\circ}$, respectively. Overall excellent agreement with the analytic description is found [27].

The analysis of experimentally obtained isofrequency contours clearly shows the influence of field-dependent anisotropy of spin-wave propagation on the interference pattern of SWs diffracted at the zone plate [4]. Firstly, changing the field value, the curvature of isofrequency contours changes and enables to shift focal point forward and backward with respect to the grating [see Figs. 8(d)-8(g) and Figs. 9(a)-9(d)]. Moreover, since the symmetry plane of the dispersion relation rotates together with the bias magnetic field (magnetization vector), it is possible to shift left/right the focal point [see Figs. 8(b) and 8(c) and Figs. 9(e) and 9(f)].
The excellent agreement of the experimental data with the analytical anisotropic dispersion relation shows that the focal characteristics of the zone plate can be fully captured by conventional theory. However, the magnetization landscape that is formed by the overlapping demagnetization fields of the closely spaced holes needs to be considered. Combining the anisotropic dispersion behavior with these complex magnetization landscapes results in great flexibility of focal characteristics when appropriately designed.

\section{CONCLUSION}

In summary, we have shown that spin-wave focusing can be realized by a Fresnel zone plate design that is capable of broadband operation. Here, our focusing approach results in a focal spot with $840-\mathrm{nm}$ FWHM at a $23 \%$ increase of spin-wave amplitude, overcompensating damping during propagation. Furthermore, the focal spot can be moved within a $40-\mu \mathrm{m}^{2}$ area behind the zone plate by varying the external magnetic-field magnitude and orientation.

The behavior of focus formation and shifting with applied field are explained by the field-dependent anisotropy of spin-wave propagation. Depending on the anisotropy, wavelength, and propagation direction, different focal positions are achieved.

Additionally, the experimental results were captured by micromagnetic simulations and supported by a theoretical approach, and it was shown that the spin-wave pattern depends strongly on the nonuniform magnetization landscape that is formed by the demagnetization fields induced by the holes. These nonuniformities shift the vertices of the causticlike spin-wave beams away from the hole edges. Careful analysis of the anisotropic dispersion relation and isofrequency contour applicable to this landscape allows a complete understanding of the operation of the Fresnel zone plate for spin waves.

The ability to focus spin waves and steer their focal position opens up a large number of possibilities in magnonics research, allowing any device to be placed into the focal position without limitations imposed by the focusing system. Furthermore, the nanometer-sized spin-wave illumination can easily be moved between different device positions, effectively creating a spin-wave distributor system.

\section{ACKNOWLEDGMENTS}

The authors would like to thank Michael Bechtel for support during beam times. Johannes Stigloher is gratefully acknowledged for fruitful discussions. Helmholtz Zentrum Berlin is acknowledged for allocating beam time at the BESSY II synchrotron radiation facility. Financial support by the Baden-Württemberg Stiftung in the framework of the Kompetenznetz Funktionelle Nanostrukturen is gratefully acknowledged. The work of P.G. was partially supported by the PL-Grid infrastructure and from the National Science Centre of Poland, under OPUS funding UMO2019/33/B/ST5/02013. M.K., P.G., and M.Z. have received partial financial support from the EU - Horizon2020 project MagIC Grant No. 644348. The simulations were partially performed at the Poznan Supercomputing and Networking Center (Grant No. 398). 
[1] V. V. Kruglyak and R. J. Hicken, Magnonics: Experiment to prove the concept, J. Magn. Magn. Mater. 306, 191 (2006).

[2] V. V. Kruglyak, S. O. Demokritov, and D. Grundler, Magnonics, J. Phys. D: Appl. Phys. 43, 264001 (2010).

[3] B. Lenk, H. Ulrichs, F. Garbs, and M. Munzenberg, The building blocks of magnonics, Phys. Rep. 507, 107 (2011).

[4] M. Krawczyk and D. Grundler, Review and prospects of magnonic crystals and devices with reprogrammable band structure, J. Phys.: Condens. Matter 26, 123202 (2014).

[5] T. Schwarze and D. Grundler, Magnonic crystal wave guide with large spin-wave propagation velocity in $\mathrm{CoFeB}$, Appl. Phys. Lett. 102, 222412 (2013).

[6] K. Vogt, F. Y. Fradin, J. E. Pearson, T. Sebastian, S. D. Bader, B. Hillebrands, A. Hoffmann, and H. Schultheiss, Realization of a spin-wave multiplexer, Nat. Commun. 5, 3727 (2014).

[7] O. Büttner, M. Bauer, S. O. Demokritov, B. Hillebrands, Y. S. Kivshar, V. Grimalsky, Y. Rapoport, M. P. Kostylev, B. A. Kalinikos, and A. N. Slavin, Spatial and spatiotemporal selffocusing of spin waves in garnet films observed by space- and time-resolved Brillouin light scattering, J. Appl. Phys. 87, 5088 (2000).

[8] V. E. Demidov, S. O. Demokritov, K. Rott, P. Krzysteczko, and G. Reiss, Self-focusing of spin waves in Permalloy microstripes, Appl. Phys. Lett. 91, 252504 (2007).

[9] D. Kumar and A. O. Adeyeye, Broadband and total autocollimation of spin waves using planar magnonic crystals, J. Appl. Phys. 117, 143901 (2015).

[10] V. E. Demidov, S. O. Demokritov, D. Birt, B. O'Gorman, M. Tsoi, and X. Li, Radiation of spin waves from the open end of a microscopic magnetic-film waveguide, Phys. Rev. B 80, 014429 (2009).

[11] T. Schneider, A. A. Serga, A. V. Chumak, C. W. Sandweg, S. Trudel, S. Wolff, M. P. Kostylev, V. S. Tiberkevich, A. N. Slavin, and B. Hillebrands, Nondiffractive Subwavelength Wave Beams in a Medium with Externally Controlled Anisotropy, Phys. Rev. Lett. 104, 197203 (2010).

[12] J.-V. Kim, R. L. Stamps, and R. E. Camley, Spin Wave Power Flow and Caustics in Ultrathin Ferromagnets with the Dzyaloshinskii-Moriya Interaction, Phys. Rev. Lett. 117, 197204 (2016).

[13] J. Stigloher, M. Decker, H. S. Körner, K. Tanabe, T. Moriyama, T. Taniguchi, H. Hata, M. Madami, G. Gubbiotti, K. Kobayashi, T. Ono, and C. H. Back, Snell's Law for Spin Waves, Phys. Rev. Lett. 117, 037204 (2016).

[14] J. Mulkers, B. Van Waeyenberge, and M. V. Milošević, Tunable Snell's law for spin waves in heterochiral magnetic films, Phys. Rev. B 97, 104422 (2018).

[15] K. Tanabe, R. Matsumoto, J.-i. Ohe, S. Murakami, T. Moriyama, D. Chiba, K. Kobayashi, and T. Ono, Real-time observation of Snell's law for spin waves in thin ferromagnetic films, Appl. Phys. Express 7, 053001 (2014).

[16] O. Dzyapko, I. V. Borisenko, V. E. Demidov, W. Pernice, and S. O. Demokritov, Reconfigurable heat-induced spin wave lenses, Appl. Phys. Lett. 109, 232407 (2016).

[17] N. J. Whitehead, S. A. R. Horsley, T. G. Philbin, and V. V. Kruglyak, A Luneburg lens for spin waves, Appl. Phys. Lett. 113, 212404 (2018).

[18] M. Vogel, R. Aßmann, P. Pirro, A. V. Chumak, B. Hillebrands, and G. von Freymann, Control of spin-wave propagation using magnetisation gradients, Sci. Rep. 8, 11099 (2018).
[19] P. Gruszecki and M. Krawczyk, Spin-wave beam propagation in ferromagnetic thin films with graded refractive index: Mirage effect and prospective applications, Phys. Rev. B 97, 094424 (2018).

[20] M. Zelent, M. Mailyan, V. Vashistha, P. Gruszecki, O. Y. Gorobets, Y. I. Gorobets, and M. Krawczyk, Spin wave collimation using a flat metasurface, Nanoscale 11, 9743 (2019).

[21] S. Mansfeld, J. Topp, K. Martens, J. N. Toedt, W. Hansen, D. Heitmann, and S. Mendach, Spin Wave Diffraction and Perfect Imaging of a Grating, Phys. Rev. Lett. 108, 047204 (2012).

[22] Á. Papp, W. Porod, Á. I. Csurgay, and G. Csaba, Nanoscale spectrum analyzer based on spin-wave interference, Sci. Rep. 7, 9245 (2017).

[23] G. A. Vugal'ter and I. A. Gilinskii, Magnetostatic waves (a review), Radiophys. Quantum Electron. 32, 869 (1989).

[24] A. V. G. Vashkovskii, K. V. Stal'makhov, A. V. Tyulyukin, and V. A. Radiotekh, Elektron 32, 1176 (1987).

[25] A. V. G. Vashkovskii, K. V. Stal'makhov, A. V. Tyulyukin, and V. A. Radiotekh, Elektron 31, 838 (1986).

[26] K. Keskinbora, C. Grévent, U. Eigenthaler, M. Weigand, and G. Schütz, Rapid prototyping of Fresnel zone plates via direct $\mathrm{Ga}+$ ion beam lithography for high-resolution x-ray imaging, ACS Nano 7, 9788 (2013).

[27] B. A. Kalinikos and A. N. Slavin, Theory of dipole-exchange spin wave spectrum for ferromagnetic films with mixed exchange boundary conditions, J. Phys. C: Solid State Phys. 19, 7013 (1986).

[28] R. Gieniusz, P. Gruszecki, M. Krawczyk, U. Guzowska, A. Stognij, and A. Maziewski, The switching of strong spin wave beams in patterned garnet films, Sci. Rep. 7, 8771 (2017).

[29] R. Gieniusz, H. Ulrichs, V. D. Bessonov, U. Guzowska, A. I. Stognii, and A. Maziewski, Single antidot as a passive way to create caustic spin-wave beams in yttrium iron garnet films, Appl. Phys. Lett. 102, 102409 (2013).

[30] D. G. Voelz, Computational Fourier Optics: A MATLAB tutorial (SPIE Press, Bellingham, Washington, USA, 2011).

[31] K. Keskinbora, U. T. Sanli, C. Grévent, and G. Schütz, Fabrication and X-ray testing of true kinoform lenses with high efficiencies, Proc. SPIE 9592 (2015).

[32] K. Li, M. Wojcik, and C. Jacobsen, Multislice does it all calculating the performance of nanofocusing X-ray optics, Opt. Express 25, 1831 (2017).

[33] D. Nolle, M. Weigand, P. Audehm, E. Goering, U. Wiesemann, C. Wolter, E. Nolle, and G. Schütz, Note: Unique characterization possibilities in the ultra high vacuum scanning transmission X-ray microscope (UHV-STXM) "MAXYMUS" using a rotatable permanent magnetic field up to $0.22 \mathrm{~T}$, Rev. Sci. Instrum. 83, 046112 (2012).

[34] J. Leliaert, B. V. d. Wiele, A. Vansteenkiste, L. Laurson, G. Durin, L. Dupré, and B. V. Waeyenberge, Current-driven domain wall mobility in polycrystalline Permalloy nanowires: A numerical study, J. Appl. Phys. 115, 233903 (2014).

[35] A. Vansteenkiste, J. Leliaert, M. Dvornik, M. Helsen, F. GarciaSanchez, and B. V. Waeyenberge, The design and verification of MuMax3, AIP Adv. 4, 107133 (2014).

[36] J. Mulkers, B. Van Waeyenberge, and M. V. Milošević, Effects of spatially engineered Dzyaloshinskii-Moriya interaction in ferromagnetic films, Phys. Rev. B 95, 144401 (2017).

[37] L. Exl, S. Bance, F. Reichel, T. Schrefl, H. P. Stimming, and N. J. Mauser, LaBonte's method revisited: An effective steepest 
descent method for micromagnetic energy minimization, J. Appl. Phys. 115, 17D118 (2014).

[38] G. Schütz, W. Wagner, W. Wilhelm, P. Kienle, R. Zeller, R. Frahm, and G. Materlik, Absorption of Circularly Polarized x Rays in Iron, Phys. Rev. Lett. 58, 737 (1987).

[39] F. Groß, N. Träger, J. Förster, M. Weigand, G. Schütz, and J. Gräfe, Nanoscale detection of spin wave deflection angles in permalloy, Appl. Phys. Lett. 114, 012406 (2019).

[40] See Supplemental Material at http://link.aps.org/supplemental/ 10.1103/PhysRevB.102.024420 for spin-wave movie for transmission through a hole-based Fresnel zone plate in a $50-\mathrm{nm}$
Py thin film at $3.6 \mathrm{GHz}$ with an applied in-plane bias field of $-12 \mathrm{mT}$.

[41] See Supplemental Material at http://link.aps.org/supplemental/ 10.1103/PhysRevB.102.024420 for spin-wave movie for transmission through a hole-based Fresnel zone plate in a 50-nm Py thin film at $5.7 \mathrm{GHz}$ with an applied in-plane bias field of $-18 \mathrm{mT}$.

[42] T. Sebastian, T. Brächer, P. Pirro, A. A. Serga, B. Hillebrands, T. Kubota, H. Naganuma, M. Oogane, and Y. Ando, Nonlinear Emission of Spin-Wave Caustics from an Edge Mode of a Microstructured Co_\{2\}Mn_\{0.6\}Fe_\{0.4\}Si Waveguide, Phys. Rev. Lett. 110, 067201 (2013). 